\title{
Rehabilitation of Severely Worn Dentition and Partial Edentulism by Fixed and Removable Prostheses: A Clinical Report
}

\author{
Vaibhav Deorao Kamble
}

\begin{abstract}
The esthetic and functional rehabilitation of severely worn dentition and partial edentulism is probably one of the most intellectually and technically demanding tasks faced by the restorative dentist. The severe wear of teeth and missing teeth has multiple implications in relation to loss of anterior guidance, reduction of vertical dimension and disfigurement of the occlusal plane. Hence, restoring vertical dimension without compromising occlusal, functional and esthetic requirements is utmost essential for long-term success. This clinical report describes the rehabilitation of patient with severely worn dentition and missing posterior teeth using the integration of fixed and removable prostheses. This case reports improvement in phonetics and occlusions with esthetic enhancement by restoring sufficient interocclusal space.
\end{abstract}

Keywords: Worn dentition, Partial edentulism, Vertical dimension of occlusion, Full mouth rehabilitation, Fixed and removable prostheses.

How to cite this article: Kamble VD. Rehabilitation of Severely Worn Dentition and Partial Edentulism by Fixed and Removable Prostheses: A Clinical Report. Int J Prosthodont Restor Dent 2013;3(2):57-61.

\section{Source of support: Nil}

Conflict of interest: None

\section{INTRODUCTION}

Some missing teeth can cause multiple biological, esthetic and functional complications. ${ }^{1,2}$ Due to loss of some natural teeth, relationship between teeth in the same arch and with the opposing dentition might be severely affected. ${ }^{3}$ Further rehabilitation will be complicated due to invasive treatment like intentional endodontics, orthodontics, crown lengthening procedure, and alteration of vertical dimension of occlusion (VDO). ${ }^{3}$ According to cause, tooth wear can be categorized as abrasion, erosion and attrition which occur in combination in most clinical conditions. ${ }^{4}$ The excessive teeth wear can lead to occlusal disharmony, pulpal disorders, compromised esthetics, mastication and speech. ${ }^{5}$ It is utmost essential to identify the factors causing excessive occlusal wear and to analyze loss of VDO. ${ }^{6}$ In many cases, VDO is maintained by growth of alveolar bone and eruption of teeth. Growth of alveolar bone compensates for tooth structure loss as an adaptive mechanism in severely worn dentition. Hence, VDO should not be modified without vigilant approach. Dahl et al used removable anterior occlusal device in severe localized attrition to develop sufficient interocclusal space for restoration. ${ }^{6,7}$ Nowadays the adhesive resin or an overlay splint is used. ${ }^{8-11}$ Management of severely worn dentition and partial edentulism is a complicated clinical situation in terms of difficulty in achieving an esthetically and functionally acceptable restoration as well as the cost. ${ }^{12}$ Mounted study models and diagnostic mock-up can provide valuable information for the evaluation of VDO. An occlusal splint or provisional restoration can be useful adjunct to confirm the patient's tolerance to modified VDO.$^{13}$ This clinical report describes the full mouth rehabilitation of severely worn dentition and missing posterior teeth with integration of fixed and removable prosthesis.

\section{CLINICAL REPORT}

A 56-year-old partially dentate male patient reported to the Department of Prosthodontics, Government Dental College and Hospital, Nagpur, for the treatment of severely worn dentition and missing posterior teeth. His chief complaint was difficulty in mastication due to attrition of anterior teeth and absence of posterior teeth. Medical history revealed that patient has hypertension and diabetes. Intraoral examination revealed loss of tooth structure especially in anterior teeth with sharp edges and craters in dentin (Figs 1A to C). Discrepancy was found between centric occlusion and maximum intercuspation as guided in centric relation with bimanual manipulation technique as described by Dawson. The periodontal examination revealed generalized bleeding on probing. Maxillary right second molar and mandibular left second molar were grade II mobile with furcation involvement. The radiographic examination included panoramic radiograph to evaluate the periodontal health (Fig. 2). The bone resorption in the interdental and edentulous region was found to be moderate. On the basis of clinical and radiographic examination, the patient was diagnosed to have excessive wear with loss of VDO (Turner's Category No. 1). ${ }^{3}$ According to the prosthodontic diagnostic index (PDI), patient was classified as a PDI class IV; maxillary and mandibular Kennedy class I with insufficient tooth structure and guarded prognosis for some abutments and requiring re-establishment of the occlusion with a change in the VDO. ${ }^{14} \mathrm{VDO}$ was assessed by phonetic evaluation, interocclusal space measurement and facial appearance. ${ }^{15-17}$ Following preprosthetic phase and oral 


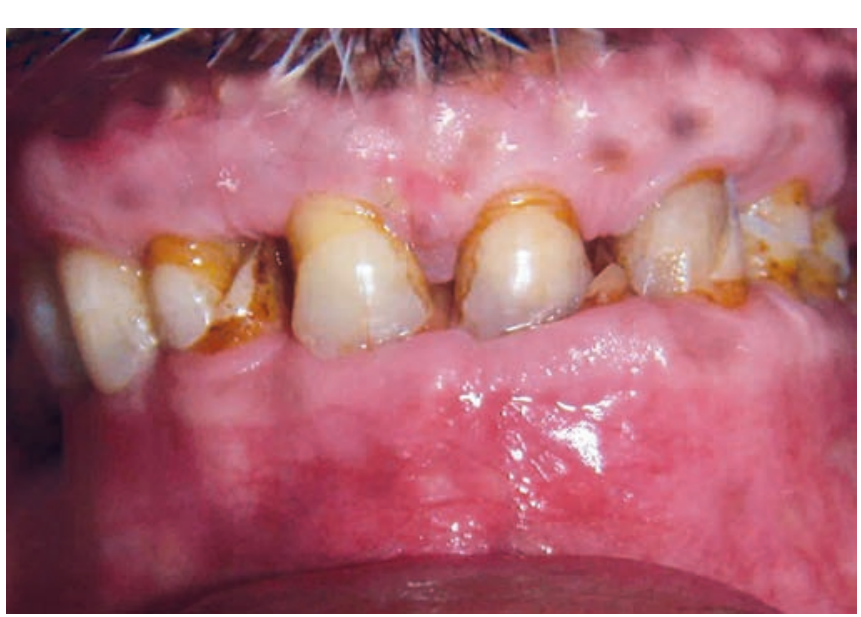

Fig. 1A: Pretreatment view of teeth in occlusion

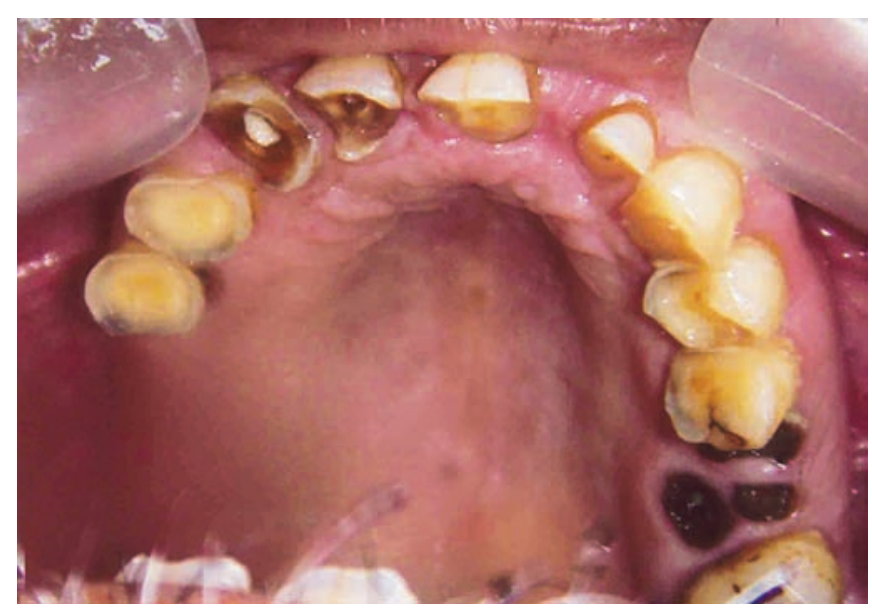

Fig. 1B: Maxillary occlusal (mirror) view

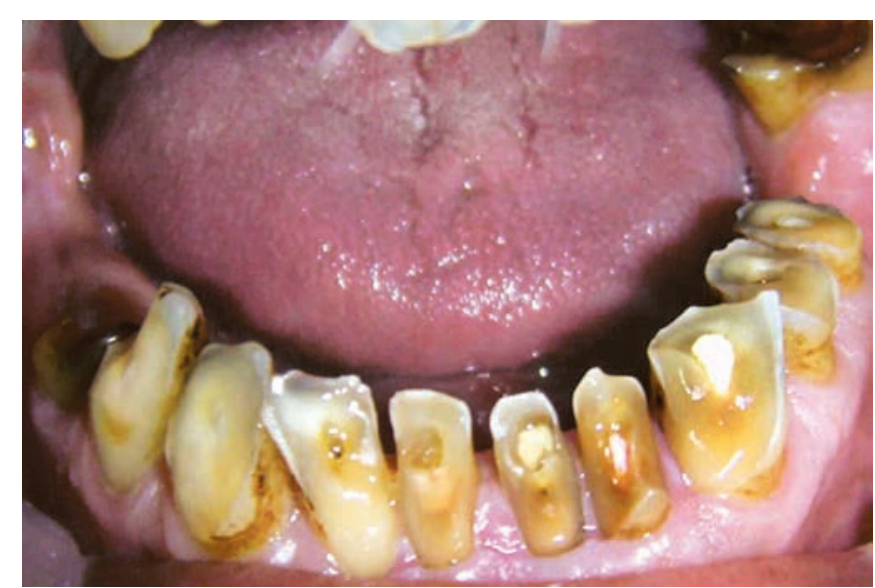

Fig. 1C: Mandibular occlusal view

prophylaxis, maxillary and mandibular impressions were made in irreversible hydrocolloid (Neocolloid, Zhermack, Rovigo, Italy). Diagnostic casts were fabricated from type III gypsum product (Kalstone; Kalabhai Karson, Mumbai, India) and mounted on a semiadjustable articulator (Whip Mix; Model 8500, Whip Mix Corp, Louisville, USA) with a face bow (Quick mount face bow; Model 8645,

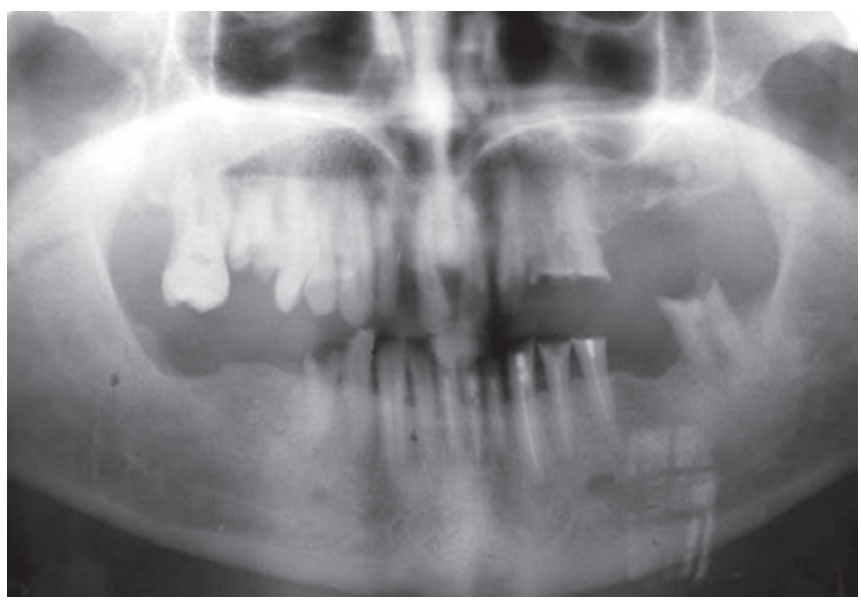

Fig. 2: Pretreatment panoramic radiograph

Whip Mix Corp, Louisville, USA) and an interocclusal record that was made with Lucia jig and polyvinyl siloxane bite registration material (EXABITE II; GC Corp., Tokyo, Japan). A mandibular occlusal splint was fabricated in acrylic resin (Travelon Clear Denture Material, Dentsply India, Gurgaon, India) with uniform occlusal contacts to permit free horizontal movement of mandible to deprogram the muscles completely. Patient was instructed to wear the occlusal splint for maximum permissible hours per day for 3 months. The occlusal splint assisted the effect of increased VDO on the temporomandibular joint (TMJ) and surrounding musculature. Improvement in speech, esthetics and comfort confirmed the patient's adaptation to the new restored VDO. The treatment plan was full mouth rehabilitation with porcelain fused to metal restorations with crown lengthening surgery and removable cast partial dentures to replace missing posterior teeth. Crown lengthening of severely worn teeth was carried out to obtain a sufficient clinical crown length. ${ }^{18}$ Increase in interocclusal space was determined $3 \mathrm{~mm}$ in the anterior teeth and 1 to $2 \mathrm{~mm}$ in the posterior teeth. Diagnostic wax-up was performed to establish group function occlusion, which helps to distribute the forces over the greatest possible area on the working side during lateral excursions of the mandible. ${ }^{19}$ Provisional fixed restorations were fabricated using a vacuum formed matrix (Drufolen H; Dreve Dentamid GmbH, Unna, Germany). Maxillary and mandibular interim RPDs were fabricated to fit with provisional restorations. The provisional restorations were cemented with temporary luting cement (Freegenol Temporary pack; GC Corp., Tokyo, Japan) and interim RPDs delivered (Fig. 3). During 3 months provisional period, the patient's functions such as mastication, TMJ discomfort, mandibular movements, muscle tenderness, speech and swallowing were evaluated. Customized anterior guide table was fabricated with acrylic resin (Rapid Repair; Dentsply India, Gurgaon, India) at modified occlusion. Final tooth 


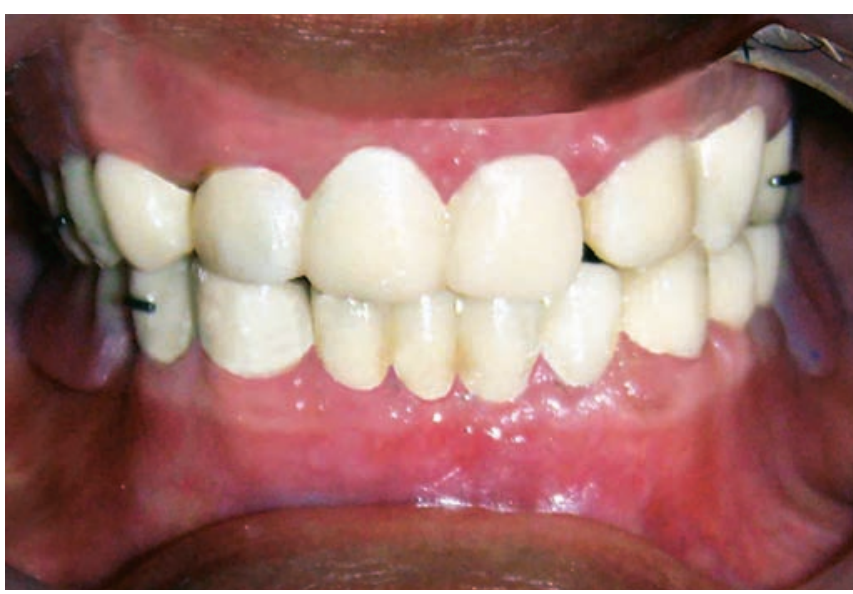

Fig. 3: Provisional restorations and interim RPD at raised VDO

preparation was performed, and definitive impressions were made with polyvinyl siloxane impression material (Exaflex \& Examix; GC America Inc, Alisip, Illinois, USA) by putty wash technique. Bite registration was recorded using provisional crown and occlusal registration material (StoneBite; Dreve Dentamid GmbH, Unna, Germany). Porcelain fused to metal restorations were fabricated and cemented with glass ionomer cement (GC Fuji I, GC Corporation, Tokyo, Japan) (Figs 4 and 5). While fabricating porcelain fused to metal restorations rest seats for maxillary and mandibular cast partial dentures were prepared in wax pattern. The functional impression on posterior alveolar ridge in both arches was made with tray attached to the RPD framework, and the altered cast was fabricated. After the adaptation of framework and the trial of waxed-up denture, the definitive prostheses were fabricated and delivered with minor occlusal adjustment in bilateral balanced occlusal scheme in opposing RPDs (Figs 6A to C). Oral hygiene instructions were given to the patient. Patient is being followed up regularly and is satisfied with prostheses. There was no complaint of any pain or discomfort in the TMJ region during 3 years of clinical service.

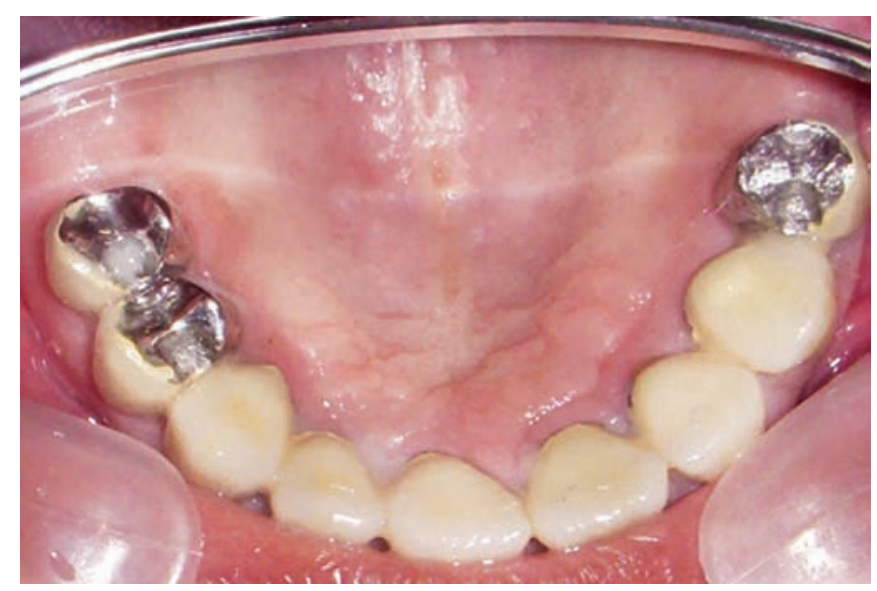

Fig. 4: Cementation of PFM restorations. Maxillary occlusal (mirror) view

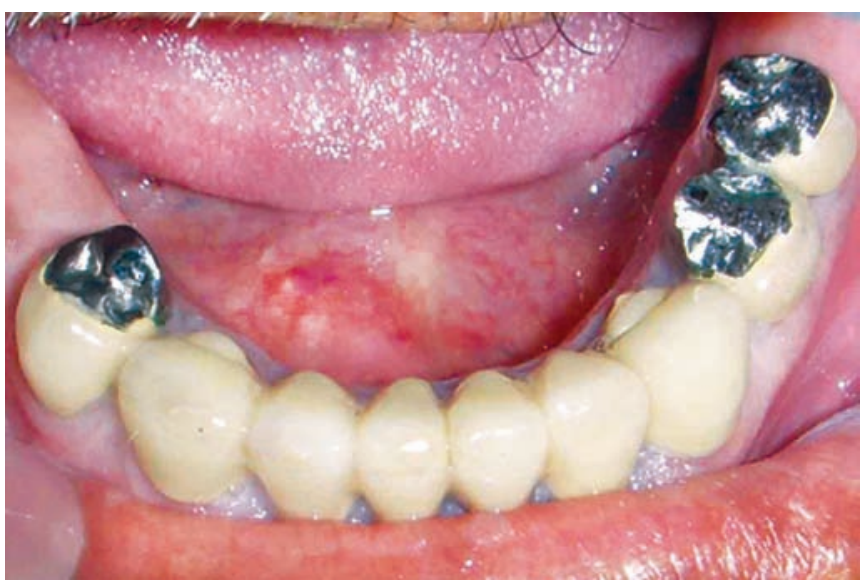

Fig. 5: Cementation of PFM restorations. Mandibular occlusal view. Note: rest seats prepared for definitive RPD

\section{DISCUSSION}

Patients requiring extensive rehabilitation present with extrusion of teeth, malpositioned teeth or periodontal problems. Severely worn dentition is common problem seen in the population and restorative challenge for dentists. Excessive wear due to any reason can adversely affect the esthetics, function and health of stomatognathic system. ${ }^{20}$ The restorative dentist also confronts the emotional problems of patients who often present with complicating conditions as a consequence of having avoided the extensive time required for proper rehabilitation. Thus, it is extremely important to shorten the period of rehabilitation as much as possible and to provide the patient with esthetic, comfortable and functional restorations that contribute to successful treatment. This clinical case illustrates the plausibility of removable partial denture rehabilitation of missing posterior teeth and severely worn dentition. Rehabilitation included a trial occlusal splint device, provisional restorations, and definitive restorations were chosen. The patient was carefully evaluated for 3 to 6 months to evaluate the patient's tolerance to altered VDO. ${ }^{21,22}$ Depending on patient's adaptive capacity, the provisional restorations and period can be modified with careful monitoring. Integration of fixed and removable prosthodontics can be a viable alternative of rehabilitating patients with moderate/severe tooth wear and partial edentulism. This approach provides a relatively simple, noninvasive and cost-effective way to achieve improvements in appearance and function of the dentition. This integrated approach also provide arch stabilization, preserve the path of insertion, maintain vertical dimension and denturesupporting structures, enhance the esthetic appearance and comfort of the patient, and may be used as a template for the definitive implant restorations. Abutment teeth are subjected to stresses under masticatory function and at rest. An RPD framework is designed maximize the support and stability by multiple rests on the remaining abutment teeth 


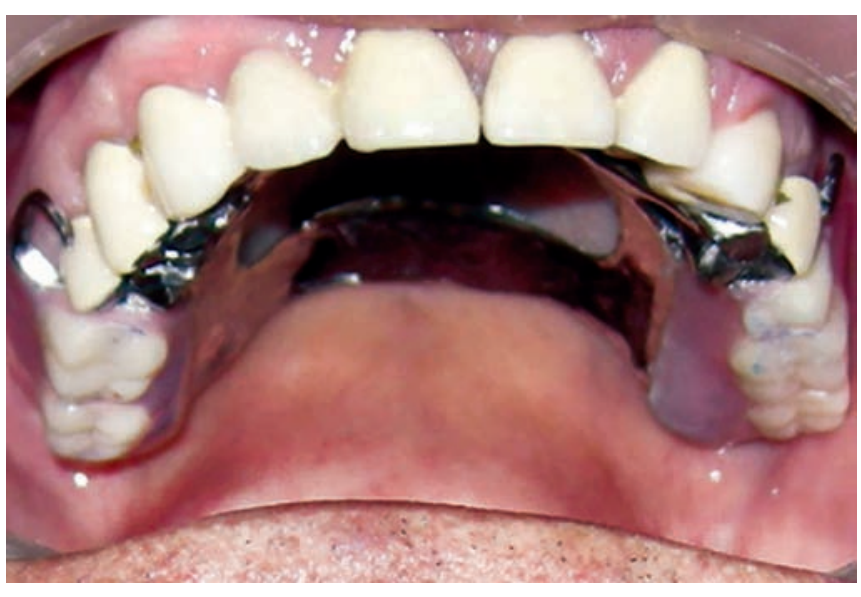

Fig. 6A: Post-treatment maxillary occlusal view

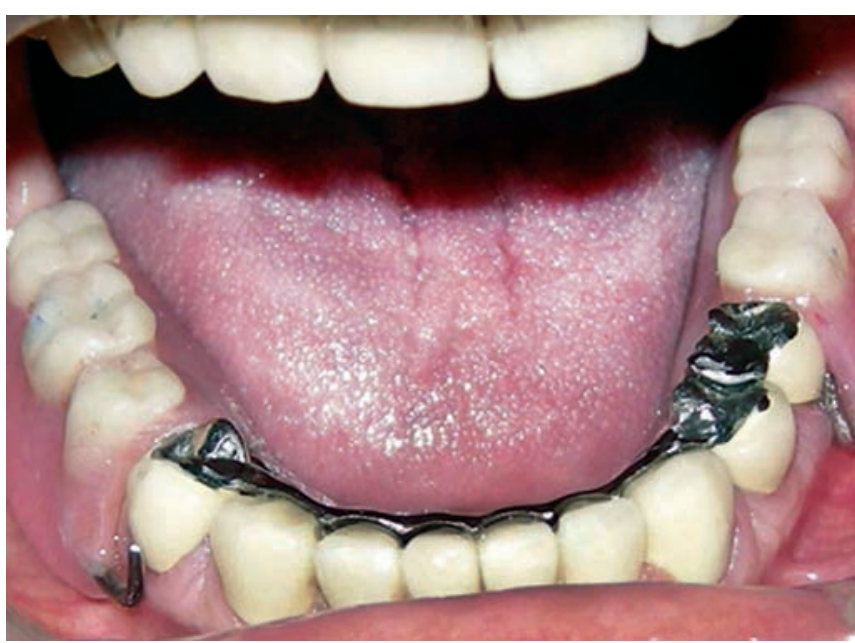

Fig. 6B: Post-treatment mandibular occlusal view

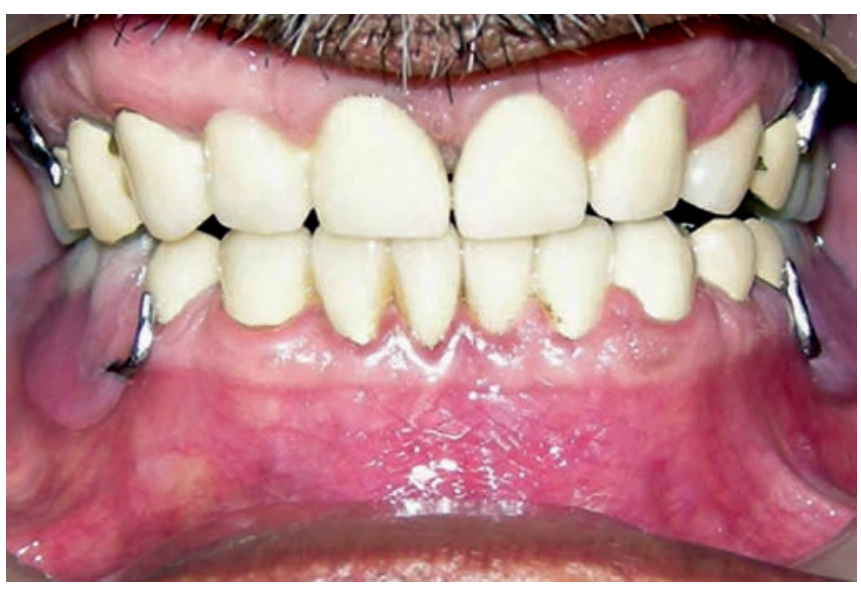

Fig. 6C: Post-treatment view of teeth in maximum intercuspation

and extending denture bases on the residual ridge during function especially in distal-extension situations. ${ }^{23,24}$ To reduce the turning movement of prosthesis, single-rooted abutment teeth are often connected to have the increased stability like multirooted teeth shifting the center of rotation to the interdental septum. The other treatment option that was discussed with the patient was the implant-supported prostheses. An implant-supported prostheses has definitely a lot of advantages as improved retention, stability and support, prevents bone loss, improved esthetics but the economical constraints and medical condition of the patient had restrained to present treatment option. This approach is simple and reversible and allows for controlling and monitoring the support and stability of the abutment teeth. The procedure described is indicated for lengthy restorations, when the dental treatment involves complex and extensive procedures such as surgical, periodontal or endodontic treatments and when fixed prostheses and removable partial prostheses are needed in the same arch. If resorption of residual ridge progresses, restorations can get exposed to excessive occlusal loads. In this patient, there was collapse of VDO and need for reorganization of occlusion. When reorganizing the occlusion, it is essential to precede restorative procedures with a period of occlusal device therapy to ensure that a stable maxilla-mandibular relationship has been achieved. An occlusal device was given to the patient to ensure his accommodation to increased VDO. A multidisciplinary approach was taken to rehabilitate the patient's mutilated dentition in an organized way. The successful integration of fixed and removable prosthodontics has resulted in accurately fitting, esthetic and functionally efficient prostheses. Because the compliance of patients in wearing free-end saddle dentures has been shown to be poor, the education on wearing RPD is necessary. ${ }^{25}$ Regular recall and maintenance visits are therefore essential to ensure the long-term success of RPDs.

\section{CONCLUSION}

In this clinical report, raising VDO using provisional prosthetic phase based on accurate diagnosis showed successful rehabilitation for severely worn dentition and partial edentulism and subsequently improved the patient's esthetics and oral function, and established favorable plane of occlusion. In patients with limited finances, low esthetic concerns, or medical limitations, this may be a viable or often the only treatment option. Irrespective of the aforementioned factors, all patients with severely worn dentition with decreased VDO and missing teeth should be presented with this treatment option.

\section{REFERENCES}

1. Davenport JC, Basker RM, Heath JR, Ralph JP, Glantz PO. The removable partial denture equation. Br Dent J 2000;189:414-424.

2. Eckert SE. Sequelae of partial edentulism. Int J Prosthodont 2007;20:356-360.

3. Turner KA, Missirlian DM. Restoration of the extremely worn dentition. J Prosthet Dent 1984;52:467-474.

4. Dawson PE. Functional occlusion: from TMJ to smile design. 1st ed. New York: Elsevier Inc.; 2008. p. 430-452. 
5. Jahangiri L, Jang S. Onlay partial denture technique for assessment of adequate occlusal vertical dimension: a clinical report. J Prosthet Dent 2002;87:1-4.

6. Dahl BL, Krogstad O, Karlsen K. An alternative treatment in cases with advanced localized attrition. J Oral Rehabil 1975;2:209-214.

7. Dahl BL, Krogstad O. Long-term observations of an increased occlusal face height obtained by a combined orthodontic/ prosthetic approach. J Oral Rehabil 1985;12:173-176.

8. Hemmings KW, Darbar UR, Vaughan S. Tooth wear treated with direct composite restorations at an increased vertical dimension: results at 30 months. J Prosthet Dent 2000;83:287-293.

9. Darbar UR, Hemmings KW. Treatment of localized anterior tooth wear with composite restorations at an increased vertical dimension. Dent Update 1997;24:72-75.

10. Sato S, Hotta TH, Pedrazzi V. Removable occlusal overlay splint in the management of tooth wear: a clinical report. J Prosthet Dent 2000;83:392-395.

11. Windchy AM, Morris JC. An alternative treatment with overlay removable partial denture: a clinical report. J Prosthet Dent 1998;79:249-253.

12. Crothers AJR. Tooth wear and facial morphology. J Dent 1998;20:333-341.

13. Hemmings KW, Howlett JA, Woodley NJ, Griffiths BM. Partial dentures for patients with advanced tooth wear. Dent Update 1995;22:52-59.

14. McGarry TJ, Nimmo A, Skiba JF, Ahlstrom RH, Smith CR, Koumjian JH, Arbree NS. Classification system for partial edentulism. J Prosthodont 2002;11:181-193.

15. Pound $\mathrm{E}$. The mandibular movements of speech and their seven related values. J South Calif Dent Soc 1966;34:435-439.
16. Silverman MM. The speaking method in measuring vertical dimension. J Prosthet Dent 1953;3:193-199.

17. Niswonger ME. The rest position of the mandible and the centric relation. J Am Dent Assoc 1934;21:1572-1576.

18. Hempton TJ, Dominici JT. Contemporary crown-lengthening therapy: a review. J Am Dent Assoc 2010;141:647-655.

19. Schuler $\mathrm{CH}$. Considerations of occlusion in fixed partial dentures. Dent Clin North Am 1959;3:175.

20. Bronstein BR. An evaluation of basic concepts in mouth rehabilitation. J Prosthet Dent 1951;1:560-569.

21. Johansson A, Johansson AK, Omar R, Carlsson GE. Rehabilitation of the worn dentition. J Oral Rehabil 2008;35: 548-566.

22. Ganddini MR, Al-Mardini M, Graser GN, Almog D. Maxillary and mandibular overlay removable partial dentures for the restoration of worn teeth. J Prosthet Dent 2004;91:210-214.

23. Frechette AR. Partial denture planning with special reference to stress distribution. J Prosthet Dent 1951;1:710-724.

24. Kratochvil FJ. Maintaining supporting structures with a removable partial prosthesis. J Prosthet Dent 1971;25:167-174.

25. Witter DJ, Van Elteren P, Kayser AF, Van Rossum GM. Oral comfort in shortened dental arches. J Oral Rehabil 1990;17: 137-143.

\section{ABOUT THE AUTHOR}

\section{Vaibhav Deorao Kamble}

Senior Lecturer, Department of Prosthodontics, VSPM's Dental College and Research Centre, Nagpur, Maharashtra, India, Phone: 9561381468, e-mail: vaibhavk056@gmail.com 